\title{
Major Histocompatibility Complex Class I in Hippocampus Promotes the Comorbid Depressive Symptoms in Cancer Induced Bone Pain via Regulating the Microglial TREM2/DAP12 Signaling
}

\section{Xiaohui Li}

Taihe Hospital, Hubei University of Medicine, Shiyan, Hubei, China https://orcid.org/0000-0001-95525253

Yifu Jia

Hubei University of Medicine

Mengyuan Xiong

Hubei University of Medicine

Minjing Peng

Hubei University of Medicine

Shanchun Su

Hubei University of Medicine

Xueqin Xu

Hubei University of Medicine

Yanqiong Wu

Hubei University of Medicine

Changbin Ke ( $\nabla$ changbinke-iap@taihehospital.com )

Hubei University of Medicine

\section{Research Article}

Keywords: Major histocompatibility complex class I, cancer induced bone pain, depression, microglia, TREM2/DAP12

Posted Date: September 7th, 2021

DOl: https://doi.org/10.21203/rs.3.rs-863805/v1

License: (c) (i) This work is licensed under a Creative Commons Attribution 4.0 International License. Read Full License 


\section{Abstract}

Pain and depression comorbidity affect the patients' both physical and mental health and quality of life seriously. The comorbid depressive symptoms in cancer pain severely affect the recognition and treatment of pain. Similarly, cancer pain patients with depression are inclined toward more despair and greater impairment. The mechanisms responsible for the comorbid depressive symptoms in cancer induced bone pain have not been fully delineated and the currently available therapeutics for this pathological pain is relatively limited. In the present study, we observed that carcinoma cells implantation induced pain and depression comorbidity resulted in the upregulation of major histocompatibility complex class I (MHC-I) in hippocampus associated with the activation of TREM2/DAP12-mediated microglial signaling pathways. These observations were reversed by a lentiviral vector harboring RNA interference sequence targeting MHC-I injected into the hippocampus of tumor bearing mice. Together, these results suggest that MHC-I involves in the cancer induced bone pain and depression comorbidity through regulating the TREM2/DAP12-mediated signals in microglia of hippocampus. Suppression of MHC-I could be a therapeutic target for cancer induced bone pain.

\section{Introduction}

Pain and depression frequently occur together in clinical, particularly for cancer patients (David Spiegel 1994). The prevalence of major depression is up to $20 \%$ in cancer patients and tends to be higher due to inadequate management of pain. Furthermore, the severity of depressive symptoms may, in turn, make pain treatment more complex and difficult for cancer patients (Novin Nikbakhsh 2014). Pain and depression also share common biological mechanism and neurotransmitter pathways, suggesting the appropriate treatment may involve in both concurrently (Kim et al. 2012; Mystakidou et al.

2007). Therefore, a better understanding of the underlying mechanism in the comorbidity of chronic pain and depression is needed for safe and specific medications research and development.

Microglia, the important immune cells in the central nervous system (CNS), can make the response to injury or infection immediately, and plays a vital role in the pathological progression of some disorders, such as Alzheimer's disease, Parkinson's disease, and chronic pain. Studies indicate that microglia implicated in initiating, developing, and sustaining state in different types of pain. Peripheral nerve injury not only results in mechanical allodynia but also causes remarkable microgliosis in the spinal cord (Clark et al. 2007). Raghavendra et al. reported that microglial inhibitor minocycline attenuated mechanical hyperalgesia and allodynia after spinal nerve transection, which was related to the suppression of microglial activation in the spinal cord (Raghavendra et al. 2003). Activated microglia have potentially exerted a powerful influence on the excitation in lamina I neurons of the spinal cord dorsal horn through various modifications of plasticity in neuronal synapses, connections and networks, eventually alterations in this pathological signal can affect the neuronal function in the brain. Several studies have revealed that peripheral nerve injury also results in microglial activation in brain regions including the thalamus, amygdala, anterior cingulate cortex, and hippocampus (Barcelon et al. 2019; Hu et al. 2020; Liu et al. 2017; Zhao et al. 2007). Dai et al has indicated the potential associations between minocycline and 
depressive-like behaviors in rats treated with carcinoma cells induced bone pain via inhibiting microglia polarization with M1/M2 phenotype changes (Dai et al. 2019). From these studies, it is obvious that the activated microglia plays a vital role in chronic pain, however, it remains unclear how microglia are activated in the brain regions and the underlying molecular mechanisms deserve to be investigated in greater depth in BCP.

MHC-I is a transmembrane protein that comprises a soluble $\beta 2$-microglobulin $(\beta 2 \mathrm{~m})$ light chain, and a peptide bound to the heavy chain. MHC-I is well known for its function in the adaptive immune response to present antigenic peptides to cytotoxic T lymphocytes. Studies on MHC-I expression have been performed in normal brain and various diseases of the CNS, and demonstrate its expression on endothelial cells and some microglia. Recent emerging evidence has documented that MHC-I has an impact on the susceptibility to the development and maintenance of pain-like behavior following peripheral nerve injury or carcinoma cells inoculation in rats (Dominguez et al. 2008; Fu et al. 2016). Here, we observed that carcinoma cells inoculation induced bone pain and depression comorbidity resulted in the upregulation of MHC-I in hippocampus. Therefore, we hypothesize that MHC-I plays a crucial role in cancer induced bone pain via activating microglia; however, the underlying mechanism remains elusive.

DNAX-activating protein of $12 \mathrm{kDa}$ (DAP12), a single-pass transmembrane adaptor protein, is primarily expressed in various myeloid cells and natural killer cells. In the CNS, DAP12 and DAP12-associated receptor, the triggering receptor expressed on myeloid cells 2 protein (TREM2), are predominantly expressed in microglia, involving in the pathogenesis of Alzheimer's disease (Jason D. Ulrich 2017; Kiialainen et al. 2005). Numerous studies reported that DAP12 and TREM2 have been implicated in the regulation of microglial phenotype and activity in a wide variety of diseases (Konishi and Kiyama 2018; Zheng et al. 2017). Masaaki et al has reported that spinal nerve injury-induced proinflammatory responses in microglia, as well as mechanical hypersensitivity were conspicuously suppressed in DAP12deficient mice. Additionally, mice without spinal nerve injury intraperitoneally injected with TREM2 agonistic antibody were elicited proinflammatory cytokine expression in microglia and pain behaviors (Kobayashi et al. 2016). The TREM2/DAP12-mediated signaling obviously implicated in the regulation of the physiological functions of microglia and exacerbates the development of pain. Accordingly, we hypothesized that MHC-I facilitated the cancer induced bone pain with depression through the activation of microglial TREM2/DAP12 signaling in hippocampus.

To verify our hypothesis, we implanted the lewis lung carcinoma cells in femoral cavity of male mice to establish the bone cancer pain (BCP) models. Nociceptive behaviors were examined by mechanical hyperalgesia to evaluate the paw withdrawal threshold and hot/cold plate tests to estimate hot- and coldevoked allodynia. Depressive-like behaviors of mice were assessed by tail suspension test and forced swim test to record the immobile time reflecting despair and hopeless behaviors. RNA interference lentivirus was used to specifically knockdown MHC-I in vivo. The mRNA level and protein expression of MHC-I, TREM2, and DAP12 were quantitated using RT-PCR and western blot analysis, respectively. Our study reveals that MHC-I upregulation in hippocampus promoted the comorbid depressive symptoms in cancer induced bone pain via activating the microglial TREM2/DAP12 signaling pathways. Therefore, the 
present study addresses the crucial role that MHC-I plays in the microglial phenotype leading to cancer induced bone pain as well as pain induced depressive-like behavior, and offered relevant evidence that the microglial TREM2/DAP12-mediated signaling pathway, is also critical for involving in chronic pain.

\section{Materials And Methods}

\section{Animals}

All experiments were approved and performed accordance to the Animal Care and Use Committee of Hubei University of Medicine (Hubei, China). Male C57BL6/J mice were provided by the Institute of Laboratory Animal Science, Hubei University of Medicine. Mice were randomly divided into four groups that are sham, BCP, BCP + Con-Iv, and BCP + RNAi-Iv. Nontargeting control lentivirus (Con-Iv) or $\beta 2 \mathrm{~m}$ RNA interference lentivirus (RNAi-Iv) were accurately injected into the hippocampus according to the coodinates of the mice received carcinoma cells, respectively. Animals were kept under an autocontrolled environment of $12 \mathrm{~h}$ light/dark cycle with food and water provided ad libitum.

\section{Construction of Recombined MHC-I RNAi-Lentivirus}

We selected $\beta 2 \mathrm{~m}$ as the siRNA target to specifically knockdown MHC-I expression according to the previous published reports (Elmer et al. 2013; Fu et al. 2016). The vectors GV248 (hU6-MCS-UbiquitinEGFP-IRES-puromycin) and $\beta 2 \mathrm{~m}$ gene (GenBank accession number NM_009735) were performed by GeneChem Co. Ltd (Shanghai, China). The control lentivirus was similar to $\beta 2 m$ RNAi-lentivirus except for expressing shRNAs. The viral titre of lentivirus we used was $3 \times 10^{8} \mathrm{TU} / \mathrm{ml}$.

\section{Preparation of the Lewis Lung Carcinoma (LLC) Ccells}

Murine LLC line LL/2 (LLC1) (CRL-1642) cells were cultured in Dulbecco's minimum essential mediumhigh glucose (DMEM-high glucose, Gibco, Thermo Fisher Scientific) added with $10 \%$ fetal bovine serum (Gibco) and then placed in $5 \% \mathrm{CO}_{2} / 95 \% \mathrm{O}_{2}$ at $37^{\circ} \mathrm{C}$. Cells were harvested by trypsinization with $0.05 \%$ trypsin-EDTA (Gibco) and settled after centrifugation at $1000 \mathrm{rpm}$ for $5 \mathrm{~min}$. Then the precipitated tumor cells were resuspended with phosphate buffered saline (PBS) and diluted to a final concentration $\left(1 \times 10^{7}\right.$ cells/ml) for the BCP model established.

\section{BCP Model Establishment}

BCP model was established as the previous published reports (Honore and Mantyh 2000; Wang et al. 2020). Briefly, under the isoflurane (3\% induction and $2 \%$ maintenance) anesthesia, the right leg was shaved and performed with $0.5 \%$ iodophor, and then a superficial incision was made on the skin overlying the patella. The tibia was discreetly exposed and drilled by a 23-gauge needle, then tumor cells $(5 \mu \mathrm{l}$, $2 \times 10^{5}$ cells $/ \mathrm{ml}$ ) was injected into the intramedullary canal of the bone by a $10 \mu \mathrm{l}$ microinjector. For the sham group, the boiled carcinoma cells were prepared in the same final concentration for injection.

\section{Microinjection of Lentivirus in the Hippocampus}


Animals were anesthetized by intraperitoneal injection of $2 \%$ phenobarbital sodium and placed on a stereotaxic apparatus (DW-2000, Chengdu Techman Software Co.,Ltd, China). An ophthalmic ointment application could be to keep the corneal lubricated. The scalp was shaved, $0.5 \%$ iodophor applied, and a midline incision made to expose the cranium. Then the cranium was drilled bilaterally with a dental drill to create burr holes following the coordinates for hippocampus: AP $\pm 2.0 \mathrm{~mm}, \mathrm{ML} \pm 1.6 \mathrm{~mm}$, DV -2.6 $\mathrm{mm}$. Virus was loaded with standard glass capillaries (Sutter Instrument) and infused at a rate of 300 $\mathrm{nl} / \mathrm{min}$ by a microsyringe pump (Longer Pump, TJ-1A). The injection needle was left for an additional 5$10 \mathrm{~min}$ and then slowly withdrawn. The incision was stitched and animals were allowed to recover over a heating pad.

\section{Nociceptive Tests}

\section{Mechanical Hyperalgesia}

Mechanical allodynia of all animals were evaluated with a dynamic plantar esthesiometer (Ugo Basile, Comerio, Italy) to record paw withdrawal threshold (PWT). Measurements were performed on day $0,7,14$, and 21 after surgery. Mice were placed individual in perspex box with a wire mesh at the bottom in a quiet environment for $30 \mathrm{~min}$. A perpendicular metal filament with $0.5 \mathrm{~mm}$ diameter was raised to the plantar surface of the ipsilateral hind paw of mice, and the force was continuous enhanced until the hindlimb was withdrawn. The final mechanical force threshold on the esthesiometer was recorded as the PWT of the mouse. Each mouse was tested three times at 10-min intervals, and the mean of three values was used to statistically analyze PWT of the mice.

\section{Hot/Cold Plate Test}

Mice were placed on a hot/cold-plate (Ugo Basile, Comerio, Italy) for $50{ }^{\circ} \mathrm{C}$ and $4{ }^{\circ} \mathrm{C}$, respectively. The latency to first lift the right hind paw during $90 \mathrm{~s}$ in hot plate test and $120 \mathrm{~s}$ in cold plate test period were recorded. Values were averaged across two separate trials for each mouse at an interval of $30 \mathrm{~min}$.

\section{Assessment of Depressive-Like Behaviors}

\section{Forced Swim Test}

Forced swim test is widely used for assessment depressive-like behavior in rodents. Each mouse was forced to swim for $6 \mathrm{~min}$ in a cylindrical plastic container $(45 \mathrm{~cm}$ in height and $20 \mathrm{~cm}$ in diameter) filled with $30 \mathrm{~cm}$ in depth of water $\left(25 \pm 1^{\circ} \mathrm{C}\right)$. The last $5 \mathrm{~min}$ of immobile time were recorded as a reflection of despair and hopeless behavior of the mouse.

\section{Tail Suspension Test}

The mouse's tail at $1 \mathrm{~cm}$ from the tip was taped to a table about $50 \mathrm{~cm}$ above the surface and tested for 6 min. The total duration of immobility was measured in the last $5 \mathrm{~min}$. Mice were considered immobile when they passive swaying and completely motionless. 


\section{Immunohistochemistry}

Under $2 \%$ pentobarbital sodium anesthesia, mice were transcardially perfused with PBS followed by cold $4 \%$ paraformaldehyde (PFA, Sigma). The brain was quickly removed, postfixed within the same fixative at $4{ }^{\circ} \mathrm{C}$ overnight, and then disposed with $30 \%$ sucrose in PBS for $48 \mathrm{~h}$ at $4{ }^{\circ} \mathrm{C}$. Brain was embedded with frozen section medium (O.C.T compound, SAKURA) and sectioned on a freezing microtome (CM1950, Leica, Germany) at $30 \mu \mathrm{m}$ in coronal planes. Slices were blocked with $5 \%$ donkey serum in PBS for 10 min at room tempreture after PBS washing and incubated with rabbit/mouse anti MHC-I antibody (1:50, Proteintech, 15240-1-AP, 66013-1-Ig), rabbit anti Iba-1 antibody (1:500, Wako, 019-19741), rabbit anti DAP12 antibody (1:200, LSBio, LS-B9453-50), and sheep anti TREM2 antibody (1:50, R\&D, AF1729) overnight at $4{ }^{\circ} \mathrm{C}$. After washing with PBS three times the next day, slices were incubated with Alexa fluorescent conjugated secondary antibodies (711-605-152, 706-545-148, Jackson ImmunoResearch, US and ab150177, abcam, UK) in darkness for $40 \mathrm{~min}$ at $37^{\circ} \mathrm{C}$. Slices were captured with a laser scanning confocal microscope (Leica TCS SP8, Wetzlar, Germany). For the microglia cell count in hippocampus, images were examined from three independent experiments of three mice per group under the same conditions and then performing statistics and analysis.

\section{RT-PCR}

Mice were euthanized after the behavioral tests, and then hippocampus were quickly removed. Total RNA was extracted from the hippocampus followed by Trizol reagent (Invitrogen, 15596026) and cDNA was synthesized using reverse transcribed kit according to the manufacturer's instructions (Takara, Japan). The targeted genes expression was measured by quantitative real time PCR on a SYBR Green qPCR Master Mix reagent system (Takara, Japan). The primer sequences of forward (F) and reverse (R) are synthesized by Sangon Biotech (Shanghai, China) and shown as follows: glyceraldehyde 3phosphate dehydrogenase (GAPDH):

F: 5'-GTGAAGGTCGGTGTGAAC-3',

R: 5'-TGAGTGGAGTCATACTGGAA-3';

MHC-I: F: 5'-GAACTGCTACGTAACACAGTTC-3',

R: 5'-GTATGTATCAGTCTCAGTGGGG-3';

TREM2: F: 5'-GGAACCGTCACCATCACTCT-3',

R: 5'-ATGCTGGCTGCAAGAAACTT-3';

DAP12: F: 5'-GATTGCCCTGGCTGTGTACT-3',

R: 5'-CTGGTCTCTGACCCTGAAGC-3'. 
Animals were euthanized after the behavioral tests, and then hippocampus were separated and put in icechilled radio-immunoprecipitation assay (RIPA) lysis buffer. The supernatant was extracted from hippocampal tissues after homogenization and disintegration. Protein concentration was detected by the BCA protein assay kit (P0012, Beyotime Biotechnology, Shanghai, China). The denatured protein samples $(30 \mu \mathrm{g})$ were separated by $10 \%$ or $12 \%$ sodium dodecyl sulfate polyacrylamide gel electrophoresis. Proteins were transferred to Immobilon-P PVDF membranes (IPVH00010, Millipore), and then membranes were blocked with 5\% nonfat dry milk diluted in Tris-buffered saline with Tween 20 (TBST) for $90 \mathrm{~min}$ at room temperature (RT). Membranes were incubated with mouse anti-a-tubulin (1:5000, Sigma, T5168), rabbit anti MHC-I (1:1000, Proteintech, 15240-1-AP), rabbit anti DAP12 (1:1000, LSBio, LS-B9453-50), and sheep anti TREM2 (1:2000, R\&D, AF1729) overnight at $4{ }^{\circ} \mathrm{C}$. Then membranes were washed with TBST, and probed with anti-mouse, anti-rabbit, and anti-sheep secondary antibody conjugated with horseradish peroxidase (1:5000, Cell Signaling Technology, 7076; 1:5000, Cell Signaling Technology, 7074; 1:5000, Sigma, A3415) at RT for 90 min. The protein bands were detected by enhanced chemiluminescence (WBKLS0500, Millipore) after TBST washed and analyzed with Image Lab software (Bio-Rad).

\section{Statistical Analyses}

GraphPad Prism 8.0 software (La Jolla, USA) was applied to perform statistical analysis. All data were shown as mean \pm S.E.M. T-tests and one-way analysis of variance (ANOVA) followed by post hoc Bonferroni's test were performed to compare the differences between two groups and among multiple groups. $P<0.05$ was identified as statistically significant. Sample size for each experiment is presented in the figure legends.

\section{Results}

\section{The Levels of MHC-I Were Upregulated in Hippocampus of BCP Mice}

We established cancer induced bone pain model in mice through femoral inoculation of LLC1 cells. Immunofluorescence staining demonstrated that MHC-I level was significantly enhanced in CA1, CA3, and dentate gyrus (DG) regions of hippocampus in BCP mice (Fig. 1a). BCP usually develops along with aggravated bone destruction. Therefore, we adopted RT-PCR and western blot analysis to examine the mRNA level and protein expression of MHC-I in hippocampus of BCP mice at different time points. The results showed that mRNA level and protein expression of MHC-I were all increased from day 7 to 21 after tumor cells inoculated compared to the expression in the mice of sham group (Fig. $1 \mathrm{~b}$ and c).

\section{MHC-I Knockdown Prevented the Nociceptive- and Depressive-Like Behaviors of BCP Mice}

Cancer pain is often accompanied by depression symptoms. To confirm whether MHC-I was implicated in the development and persistence of BCP and comorbid depression symptoms, RNAi lentivirus were 
targeted to knockdown the expression of MHC-I and a series of behavioral experiments were used to examine the pain threshold and depressive behaviors of all mice. We successfully injected the control lentivirus and $\mathrm{MHC}-\mathrm{I}$ targeted interference lentivirus into the hippocampus of BCP mice by immunofluorescence staining. The results showed that the expression of MHC-1 was downregulated in hippocampus of cancer bearing mice treated with MHC-1 interference lentivirus (Fig. 2a). RT-PCR and western blot analysis were applied to verify the mRNA level and protein expression of MHC-I in BCP mice received the interference lentivirus. The results revealed that the mRNA level and protein expression of MHC-I were markedly decreased in BCP mice that injected interference lentivirus to about the BCP mice treated with control lentivirus (Fig. $2 \mathrm{~b}$ and c). Behavior test for assessing mechanical sensitivity were performed on day $0,7,14$, and 21 after LLC1 inoculation. On day 0 before tumor inoculation, there was no significant difference on PWT among all mice from four groups. On day 7 after tumor cell inoculation, BCP mice displayed notably decreased mechanical allodynia threshold in hind paws compared to the mice in sham group and lasts up to day 21, suggesting a sustained mechanical pain after tumor cell inoculation. MHC-I knockdown elevated the PWT in BCP mice received MHC-I targeted interference lentivirus compared to the BCP mice injected with control lentivirus (Fig. 2d). Cold sensitivity and hot sensitivity were performed on day 21 after surgery. In BCP mice, cancer induced cold allodynia and heat hyperalgesia were all attenuated on day 21 . However, MHC-I knockdown increased the latency of mice hind paw away from the cold plate and hot plate (Fig. 2e and f). In the forced swim test and tail suspension test, cancer-bearing mice spent significantly longer time immobility than the sham mice did. Notably, inhibiting the expression of MHC-I in BCP mice, the shorter time immobility was spent than BCP mice treated with control lentivirus (Fig. $2 \mathrm{~g}$ and $\mathrm{h}$ ). Taken together, these results demonstrated that cancer-bearing mice presented overt symptoms of pain and depression, and MHC-I knockdown prevented these behaviors.

\section{MHC-I Knockdown Inhibited the Activation of Microglia in Hippocampus of BCP Mice}

Previous studies reported that MHC-I was expressed by some glial cells in the mature rodent of CNS (Battye et al. 1984), and microglia may increase the expression of MHC-I antigens following CNS lesions (Moffett and Paden 1994). To identify whether MHC-I knockdown restrained the activation of microglia in hippocampus of BCP mice, we performed immunofluorescence staining to label Iba-1 (microglia marker) in hippocampus of BCP mice. The results suggested that a large number of microglia were activated in CA1, CA3, and DG regions of hippocampus in BCP mice (Fig. 3a). We confirmed that MHC-I was colocalized with Iba-1 in hippocampus (Fig. 3b). In addition, MHC-I knockdown by treated with interference lentivirus evidently suppressed the activation of microglia in CA1, CA3, and DG regions of hippocampus (Fig. 3C). We also analyzed and counted the microglial cells in CA1, CA3 and DG regions of each group, and the results indicated that cancer-bearing mice induced robust activation of microglia in hippocampus, and interfering with MHC-I expression decreased microglial activation in hippocampus (Fig. 3d). Taken together, these results suggested that MHC-I in hippocampus promoted the comorbid depressive symptoms in cancer induced bone pain may be related to the over-activated microglia.

\section{MHC-I Knockdown Suppressed TREM2/DAP12 Signaling in Hippocampus of BCP Mice}


It has been reported that TREM2/DAP12-mediated signaling in microglia is activated in response to spinal nerve injury induced pain (Kobayashi et al. 2016). To confirm the functional involvement of TREM2 and DAP12 in cancer induced bone pain, we examined the expression of TREM2 and DAP12 in hippocampus of BCP mice by PT-PCR and western blot analysis, respectively. The double immunofluorescence staining of MHC-I together with DAP12 showed that MHC-I partly colocalized with DAP12 (Fig. 4a). Additionally, RT-PCR and western blot analysis were applied to verify the mRNA level and protein expression of TREM2 and DAP12 in BCP mice received the interference lentivirus. The results demonstrated that the mRNA level and protein expression of TREM2 and DAP12 were enhanced in cancer bearing mice, which markedly decreased in $\mathrm{BCP}$ mice that injected with $\mathrm{MHC}-1$ interference lentivirus to about the BCP mice treated with control lentivirus (Fig. 4 b and c).

\section{Discussion}

Our present results have illustrated that mice implanted with carcinoma cells induced bone pain display distinct vulnerability to emotional disorder (such as depression), which is likely mediated partly by enhanced function of MHC-I in hippocampus, thereby causing activated microglial TREM2/DAP12 signaling pathways. Knockdown of MHC-I with a lentiviral vector harboring RNA interference sequence prevented the development of nociceptive- and depressive-like behaviors, and reduced the microglial activation as well as attenuated the expression of TREM2 and DAP12. This may represent a potential molecular mechanism underlying the cancer induced bone pain vulnerability to emotional disorder.

Clinical researches have indicated that chronic pain patients are accompanied with comorbid emotional disorders, such as anxiety and depression, which may be further aggravate a pain condition without effective management. Thus, anxiolytic and antidepressant drugs have been widely applied for patients with chronic pain treatment, although with the limited value (MATSUZAWA-YANAGIDA et al. 2008; Wiffen 2007). Recently, several preclinical studies have suggested that chronic sensory pain models in rodents can induce anxiety- and depressive-like behaviors (MATSUZAWA-YANAGIDA et al. 2008; Wiffen 2007). The affective component of pain to be induced needs at least 3 weeks or more in different types of animal models of chronic pain (Yin et al. 2020; Zhou et al. 2019). In our present study, we established the bone cancer pain model by implanting with lung cancer cells into the femoral cavity of male mice and observed a persistent pain threshold decrease from days 7 to 21 in nociceptive tests and verified a depressive-like behavior at 21 days. We did not observe the anxiety symptoms in mice of cancer cell treated in the open field experiment and elevated plus-maze test, which might be due to the effect of tumor bearing on the hind limbs led to the mice being less active (data not shown). However, there was no difference in the exercise ability of all the tested mice in the behavior test. The behavioral results provide original evidence suggesting that carcinoma implantation into the femoral cavity induced bone pain resulted in the mechanical hyperalgesia, heat hyperalgesia, cold allodynia, and depressive-like behaviors, which are also consistent with the published studies (Yin et al. 2020; Zhou et al. 2019) and is suitable for further investigation the underlying mechanism. 
MHC-I has been shown to play vital roles in schizophrenia, brain development, synaptic plasticity, and cognition and aging (Elmer and McAllister 2012; McAllister 2014; Shatz 2009). Recent studies have suggested that MHC-I is associated with the sensory component of chronic pain at level of medullary dorsal horn (Elmer and McAllister 2012; McAllister 2014; Shatz 2009), however, whether MHC-I was related to the emotional component of chronic pain, such as depression, has not been investigated. It has been reported that induced pain vulnerability lies primarily in brain processing of affective responses, not merely in body responses to sensory pain (Cai et al. 2018; Tracey 2005). Hippocampus is well known for its role in the regulation of emotional behaviors (Hill et al. 2015; Tang et al. 2019) and involved in the modulation of nociceptive information transmission (Soleimannejad et al. 2007). Hippocampus contains a wide range of pain related neurons and receives nociception information from the spinal cord through the brainstem pain-modulation circuits (Mills et al. 2018; Wang et al. 2016). Thus, hippocampus may serve as a pivotal structure for integration peripheral nociceptive information and procession emotional responses. Our result showed that the mRNA level and protein expression of MHC-I were all enhanced in hippocampus of cancer bearing mice at different time points from day 7 to 21 after carcinoma inoculation. The increased level of MHC-I in hippocampus of cancer bearing mice was associated with animal responses to sensory pain and depressive-like behaviors. Knockdown of hippocampus MHC-I function inhibited pain behaviors and pain-induced negative emotion in cancer bearing animals. This is in accordance with previous studies indicating that MHC-I alleviates sensory pain induced by various pain conditions in the spinal cord (Mills et al. 2018; Wang et al. 2016). Our findings highlight the MHC-I functions in hippocampus in the regulation of different components of chronic sensory pain. Given the evidence that hippocampus MHC-I for processing responses to nociceptive stimulation and to emotional factors, an underlying mechanism need to be further elucidated.

An increasing body of evidence suggests that many painful conditions such as inflammation, nerve injury, and cancer often cause microglial activation in the spinal cord. Activated spinal microglia has been directly involved in the development and persistence of abnormal and exaggerated pain states (Chen et al. 2018). The spinal cord is the first relay for the nociceptive information transmission in the CNS. Nociceptive signals initiated in peripheral sensory neurons enter the spinal cord dorsal horn and then is transmitted to the brain. In addition, many recent researches have demonstrated that microglia is also activated in brain areas in cancer induced bone pain and neuropathic pain models, contributing to the sensory and emotional components under the condition (Chen et al. 2018). Here we confirm that MHC-I was colocalized with Iba-1 in hippocampus. We also report a plenty of microglial activation in CA1, CA3, and DG regions of hippocampus of cancer bearing mice and this effect was alleviated by MHC-I knockdown. This also accords with the earlier observations, which showed that microglial activation in the hippocampus are associated with chronic constriction injury induced hyperalgesia (He et al. 2019) and intracerebroventricular application of minocycline in hippocampus alleviates depressive-like behaviors in BCP rats by inhibiting microglia activation (Dai et al. 2019). We speculate that the upregulation of $\mathrm{MHC}-\mathrm{I}$ accompanied by the microglial activation during BCP induces a reorganization of the circuit within the hippocampus that mediates the development of sensory pain and emotional 
behaviors. The localization of MHC-I on microglia and identification of MHC-I as therapeutic intervention in pain provide the impetus for developing a very promising targeted drug.

In the CNS, DAP12 and a DAP12 associated receptor, TREM2, are predominantly expressed by microglia. It has been reported that DAP12 deficiency inhibits redundant microglia activation in neuropathic pain. Moreover, intrathecal injection of agonistic TREM2 antibody caused nociceptive-like behavior in mice without nerve injury, and this pain behavior was totally abolished in DAP12-deficient mice (Kobayashi et al. 2016). Guan et al also demonstrated that DAP12 deletion by BrdU labeling has no impact on nerve injury-induced microglial activation and proliferation (Guan et al. 2016). Results from the present study showed that mRNA level and protein expression of DAP12 as well as TREM2 simultaneously increase in response to carcinoma cell inoculation. Because the changes of mRNA level and protein expression in the hippocampus after BCP were equivalent to those of microglial activation numbers, the increase in DAP12 and TREM 2 mRNA and protein were due to increased number of microglia. Our results indicated that these changes were reversed after intervention with MHC-I function. Thus, we conclude that MHC-I contributed to the development and maintenance of BCP and pain induced depressive-like behavior by mediating microglial TREM2/DAP12 signaling.

In conclusion, these findings reveal a critical role for MHC-I in the development and persistence of nociceptive- and sensory pain induced depressive-like behavior during cancer bearing. The present study showed that mechanical hyperalgesia, and heat- and cold-evoked allodynia during BCP were corresponded with increased MHC-I expression within the hippocampus. Furthermore, microglia was activated in hippocampus accompanied by the upregulation of TREM2 and DAP12. MHC-I knockdown with a lentiviral vector harboring RNAi sequence prevented the nociceptive- and pain induced depressivelike behavior during BCP that corresponded with a reduction in microglia associated with TREM2/DAP12 mediated signaling. These results indicate that MHC-I facilitate the development and persistence of cancer induced bone pain in hippocampus through a microglial TREM2/DAP12-driven mechanism. Accordingly, MHC-I may serve as a potential molecular target in the alleviation of comorbid depressive symptoms in cancer induced bone pain.

\section{Declarations}

\section{Ethics Approval and Consent to Participate}

The study was reviewed and approved by the Animal Care and Use Committee of Hubei University of Medicine (Hubei, China).

\section{Consent for Publication}

Not applicable.

\section{Availability of Data and Materials}


All data generated and analysed in the current study are included in the article materials. Further inquiries can be directed to the corresponding author.

\section{Competing Interests}

All authors have declared that no conflict of interests exists.

\section{Funding}

This work was supported by the National Natural Science Foundation of China (81971060), the Project for Education Department of Hubei Province (B2020109), the Health Commission of Hubei Province scientific research project (WJ2021F036), and the Natural Science Foundation of Hubei Province of China (2020CFB342).

\section{Authors' Contributions}

Changbin Ke: Conceptualization, Supervision, and Project administration; Yanqiong Wu: Conceptualization; Xiaohui Li: Methodology, Formal analysis, Investigation, and Writing-original draft; Yifu Jia: Methodology; Mengyaun Xiong: Methodology; Minjing Peng: Methodology; Shanchun Su: Validation, and Xueqin Xu: Validation. All authors contributed to the article and approved the submitted version.

\section{Acknowledgements}

Not Applicable.

\section{References}

1. Barcelon EE, Cho W, Jun SB, Lee SJ (2019) Brain microglial activation in chronic pain-associated affective disorder. Frontiers in Neuroscience 13

2. Battye F, Bartlett PF, Schrader JW, Wong GHW, Clark-Lewis I (1984) Inducible expression of H-2 and la antigens on brain cells. Nature 310(5979):688-691

3. Cai Y, Wang W, Paulucci-Holthauzen A, Pan ZZ (2018) Brain circuits mediating opposing effects on emotion and pain. The Journal of Neuroscience 38(28):6340-6349

4. Chen G, Zhang Y, Qadri YJ, Serhan CN, Ji R (2018) Microglia in Pain: Detrimental and Protective Roles in Pathogenesis and Resolution of Pain. Neuron 100(6):1292-1311

5. Chiou C, Huang C, Liang Y, Tsai Y, Hsu K (2012) Impairment of long-term depression in the anterior cingulate cortex of mice with bone cancer pain. Pain 153(10):2097-2108

6. Clark AK, Gentry C, Bradbury EJ, McMahon SB, Malcangio M (2007) Role of spinal microglia in rat models of peripheral nerve injury and inflammation. Eur J Pain 11(2):223-230

7. Dai J, Ding Z, Zhang J, Xu W, Guo Q, Zou W et al (2019) Minocycline relieves depressive-like behaviors in rats with bone cancer pain by inhibiting microglia activation in hippocampus. 
Anesthesia Analgesia 129(6):1733-1741

8. Spiegel D, SSCK (1994) Pain and depression in patients with cancer. Cancer 74(9):2570-2578

9. Dimitrov EL, Tsuda MC, Cameron HA, Usdin TB (2014) Anxiety- and depression-like behavior and impaired neurogenesis evoked by peripheral neuropathy persist following resolution of prolonged tactile hypersensitivity. J Neurosci 34(37):12304-12312

10. Dominguez CA, Lidman O, Hao J, Diez M, Tuncel J, Olsson T et al (2008) Genetic analysis of neuropathic pain-like behavior following peripheral nerve injury suggests a role of the major histocompatibility complex in development of allodynia. Pain 136(3):313-319

11. Elmer BM, Estes ML, Barrow SL, McAllister AK (2013) MHCl requires MEF2 transcription factors to negatively regulate synapse density during development and in disease. J Neurosci 33(34):1379113804

12. Elmer BM, McAllister AK (2012) Major histocompatibility complex class I proteins in brain development and plasticity. Trends Neurosci 35(11):660-670

13. Fu Q, Shi D, Zhou Y, Zheng H, Xiang H, Tian X et al (2016) MHC-I promotes apoptosis of GABAergic interneurons in the spinal dorsal horn and contributes to cancer induced bone pain. Experimental Neurology 28612-20

14. Guan Z, Kuhn JA, Wang X, Colquitt B, Solorzano C, Vaman S et al (2016) Injured sensory neuronderived CSF1 induces microglial proliferation and DAP12-dependent pain. Nat Neurosci 19(1):94101

15. Akiyama H, SIPL (1998) Major histocompatibility complex antigen expression on rat microglia following epidural kainic acid lesions. J Neurosci Res 2(20):147-157

16. Hansen DV, Hanson JE, Sheng M (2018) Microglia in Alzheimer's disease. J Cell Biol 217(2):459-472

17. He L, Xu R, Chen Y, Liu X, Pan Y, Cao S et al (2019) Intra-CA1 Administration of Minocycline Alters the Expression of Inflammation-Related Genes in Hippocampus of CCI Rats. Frontiers in Molecular Neuroscience 12

18. Hill AS, Sahay A, Hen R (2015) Increasing adult hippocampal neurogenesis is sufficient to reduce anxiety and depression-like behaviors. Neuropsychopharmacology (New York, N.Y.) 40(10):23682378

19. Honore P, Mantyh PW (2000) Bone cancer pain: from mechanism to model to therapy. Pain Med 1(4):303-309

20. Hu X, Liu Y, Wu J, Liu Y, Liu W, Chen J et al (2020) Inhibition of P2X7R in the amygdala ameliorates symptoms of neuropathic pain after spared nerve injury in rats. Brain, Behavior, and Immunity 88507514

21. Inoue K, Tsuda M (2018) Microglia in neuropathic pain: cellular and molecular mechanisms and therapeutic potential. Nat Rev Neurosci 19(3):138-152

22. Jason D, Ulrich TKUM (2017) Elucidating the role of TREM2 in Alzheimer's disease. Neuron 2(94):237-248 
23. Kiialainen A, Hovanes K, Paloneva J, Kopra O, Peltonen L (2005) Dap12 and Trem2, molecules involved in innate immunity and neurodegeneration, are co-expressed in the CNS. Neurobiology of Disease 18(2):314-322

24. Kim H, Barsevick AM, Fang CY, Miaskowski C (2012) Common biological pathways underlying the psychoneurological symptom cluster in cancer patients. Cancer Nurs 35(6):E1-E20

25. Kobayashi M, Konishi H, Sayo A, Takai T, Kiyama H (2016) TREM2/DAP12 signal elicits proinflammatory response in microglia and exacerbates neuropathic pain. J Neurosci 36(43):1113811150

26. Konishi H, Kiyama H (2018) Microglial TREM2/DAP12 signaling: a double-edged sword in neural diseases. Front Cell Neurosci 12:206

27. Kremer M, Becker LJ, Barrot M, Yalcin I (2021) How to study anxiety and depression in rodent models of chronic pain? Eur J Neurosci 53(1):236-270

28. Liu Y, Zhou L, Wang J, Li D, Ren W, Peng J et al (2017) TNF-a differentially regulates synaptic plasticity in the hippocampus and spinal cord by microglia-dependent mechanisms after peripheral nerve injury. The Journal of neuroscience 37(4):871-881

29. Long-Smith CM, Sullivan AM, Nolan YM (2009) The influence of microglia on the pathogenesis of Parkinson's disease. Prog Neurobiol 89(3):277-287

30. Malcangio M (2016) Microglia and chronic pain. Pain 157(5):1002-1003

31. MATSUZAWA-YANAGIDA K, NARITA M, YAMAZAKI M, SUZUKI T, NAKAJIMA M, KUZUMAKI N et al (2008) Usefulness of antidepressants for improving the neuropathic pain-like state and pain-induced anxiety through actions at different brain sites. Neuropsychopharmacology (New York, N.Y.) 33(8):1952-1965

32. McAllister AK (2014) Major histocompatibility complex I in brain development and schizophrenia. Biol Psychiat 75(4):262-268

33. Mcllwrath SL, Montera MA, Gott KM, Yang Y, Wilson CM, Selwyn R et al (2020) Manganese-enhanced MRI reveals changes within brain anxiety and aversion circuitry in rats with chronic neuropathic painand anxiety-like behaviors. Neurolmage 223117343

34. Mills EP, Di Pietro F, Alshelh Z, Peck CC, Murray GM, Vickers ER et al (2018) Brainstem pain-control circuitry connectivity in chronic neuropathic pain. The Journal of Neuroscience 38(2):465-473

35. Moffett CW, Paden CM (1994) Microglia in the rat neurohypophysis increase expression of class I major histocompatibility antigens following central nervous system injury. J Neuroimmunol 50(2):139

36. Mystakidou K, Tsilika E, Parpa E, Pathiaki M, Patiraki E, Galanos A et al (2007) Exploring the relationships between depression, hopelessness, cognitive status, pain, and spirituality in patients with advanced cancer. Arch Psychiatr Nurs 21(3):150-161

37. Novin Nikbakhsh SMSA (2014) Prevalence of depression and anxiety among cancer patients. Caspian J Intern Med 5(3):167-170 
38. Höftberger R, FAWB (2004) Expression of major histocompatibility complex class I molecules on the different cell types in multiple sclerosis lesions. Brain Pathol 1(14):43-50

39. Raghavendra V, Tanga F, DeLeo JA (2003) Inhibition of microglial activation attenuates the development but not existing hypersensitivity in a rat model of neuropathy. J Pharmacol Exp Ther 306(2):624-630

40. Shatz CJ (2009) MHC class I: an unexpected role in neuronal plasticity. Neuron 64(1):40-45

41. Soleimannejad E, Naghdi N, Semnanian S, Fathollahi Y, Kazemnejad A (2007) Antinociceptive effect of intra-hippocampal CA1 and dentate gyrus injection of MK801 and AP5 in the formalin test in adult male rats. Eur J Pharmacol 562(1-2):39-46

42. Tang M, Huang H, Li S, Zhou M, Liu Z, Huang R et al (2019) Hippocampal proteomic changes of susceptibility and resilience to depression or anxiety in a rat model of chronic mild stress. Translational Psychiatry 9(1)

43. Tracey I (2005) Nociceptive processing in the human brain. Curr Opin Neurobiol 15(4):478-487

44. Wang J, Chen R, Chen S, Gao Y, Zhang J, Feng X et al (2016) Electroacupuncture reduces the effects of acute noxious stimulation on the electrical activity of pain-related neurons in the hippocampus of control and neuropathic pain rats. Neural Plasticity 20161-11

45. Wang K, Gu Y, Liao Y, Bang S, Donnelly CR, Chen O et al (2020) PD-1 blockade inhibits osteoclast formation and murine bone cancer pain. Journal of Clinical Investigation 130(7):3603-3620

46. Wiffen TSJ (2007) Antidepressants for neuropathic pain. The cochrane database of systematic reviews 4CD005454

47. Yin W, Mei L, Sun T, Wang Y, Li J, Chen C et al (2020) A central amygdala-ventrolateral periaqueductal gray matter pathway for pain in a mouse model of depression-like behavior. Anesthesiology 132(5):1175-1196

48. Zhao P, Waxman SG, Hains BC (2007) Modulation of thalamic nociceptive processing after spinal cord injury through remote activation of thalamic microglia by cysteine cysteine chemokine ligand 21. J Neurosci 27(33):8893-8902

49. Zheng H, Jia L, Liu C, Rong Z, Zhong L, Yang L et al (2017) TREM2 promotes microglial survival by activating Wnt/ $\beta$-catenin pathway. The Journal of Neuroscience 37(7):1772-1784

50. Zhou W, Jin Y, Meng Q, Zhu X, Bai T, Tian Y et al (2019) A neural circuit for comorbid depressive symptoms in chronic pain. Nat Neurosci 22(10):1649-1658

\section{Figures}




\section{Sham}
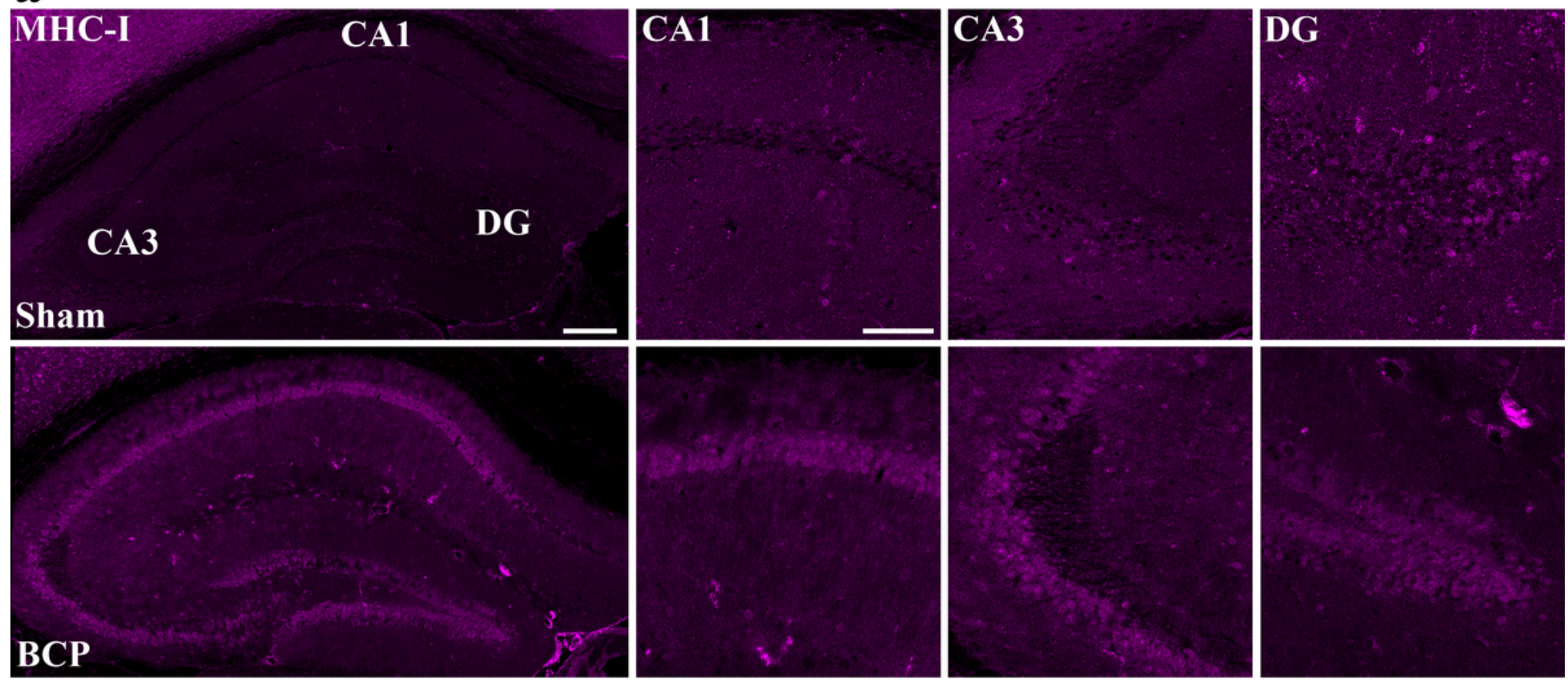

b

C
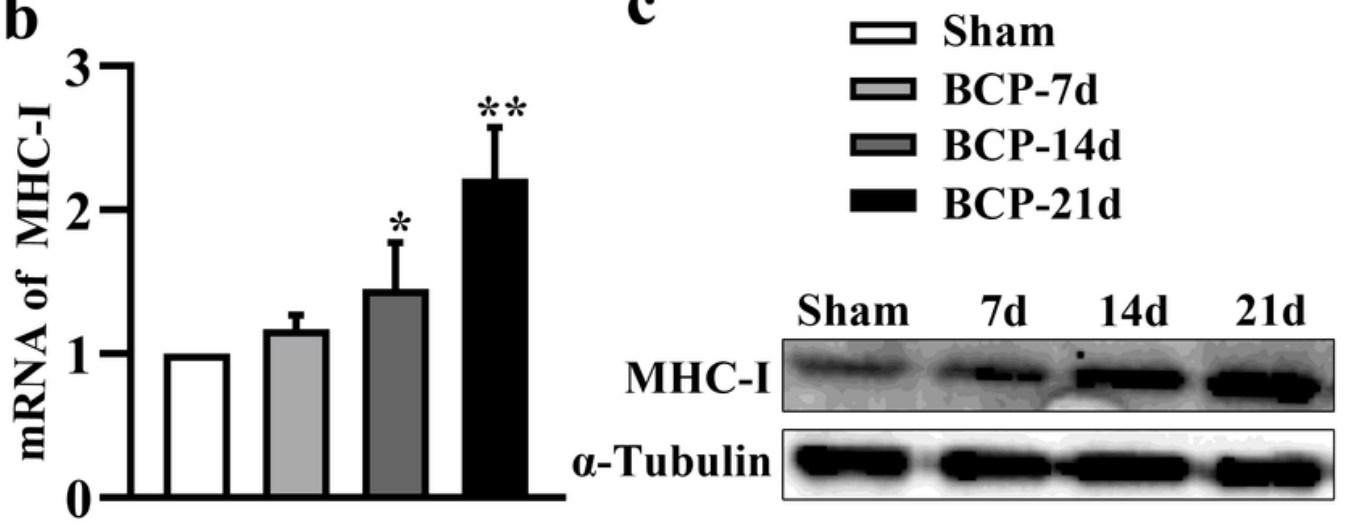

a-Tubulin

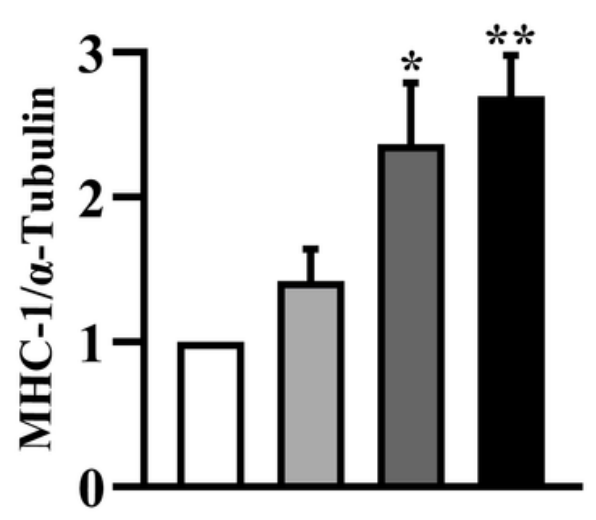

Figure 1

Cancer induced bone pain increased the expression of MHC-I in the hippocampus of mice. a.

Representative immunofluorescence images of MHC-I staining (magenta) in the CA1, CA3, and DG regions of hippocampus in sham and BCP mice. Scale bar $=200 \mu \mathrm{m}$. b. The mRNA level of MHC-I was quantitative analysed by RT-PCR. c. Representative immunoblots and quantitative analysis of MHC-I protein expression at $7 \mathrm{~d}, 14 \mathrm{~d}$, and $21 \mathrm{~d}$ after surgery, and a-Tubulin was used as an internal control. Values are normalized to the sham group and represented as mean \pm SEM. $n=4$ mice per group. $* P<$ 0.05 vs. sham group, ${ }^{\star *} P<0.01$ vs. sham group. 


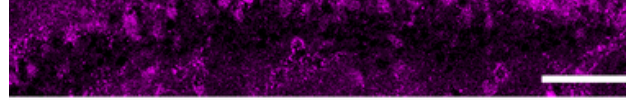

\section{MHC-I}
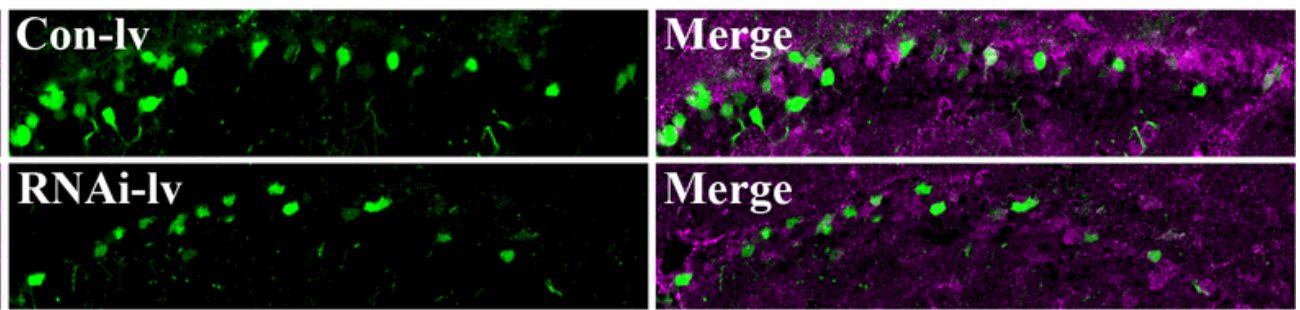

b

.
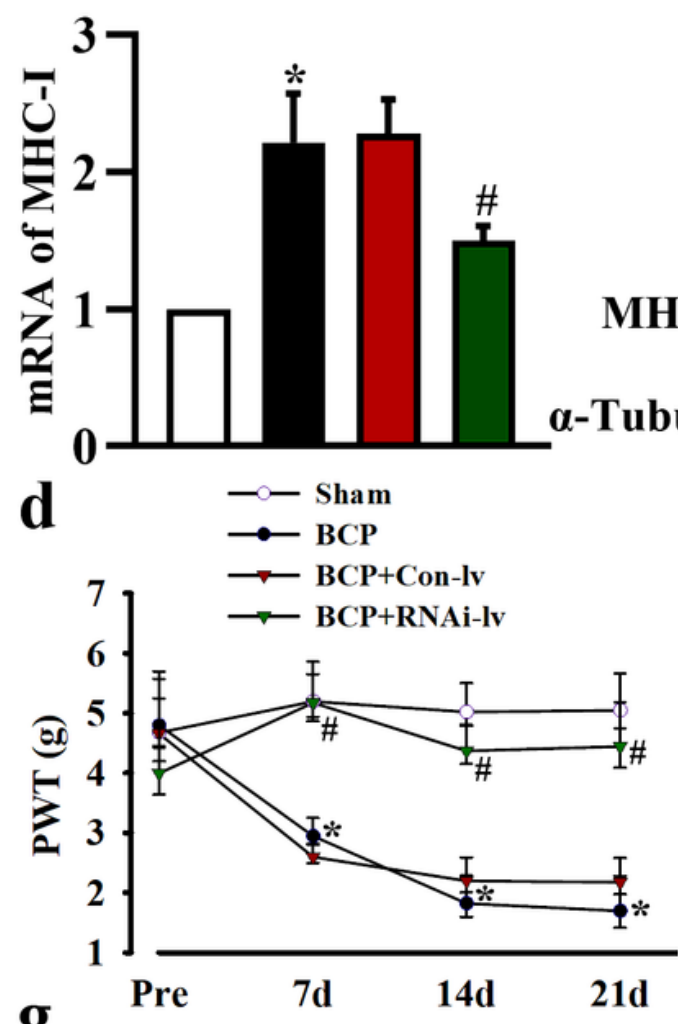

g

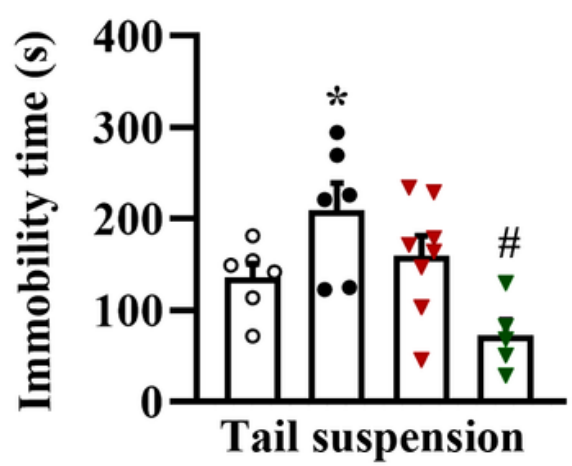

c $\square$ Sham

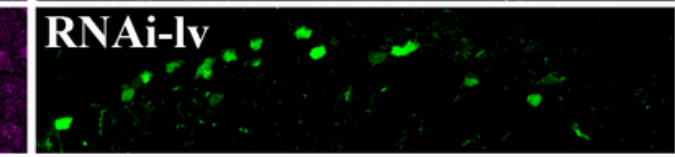

- $\mathrm{BCP}$

MHC-I

- BCP+Con-lv

BCP+RNAi-lv

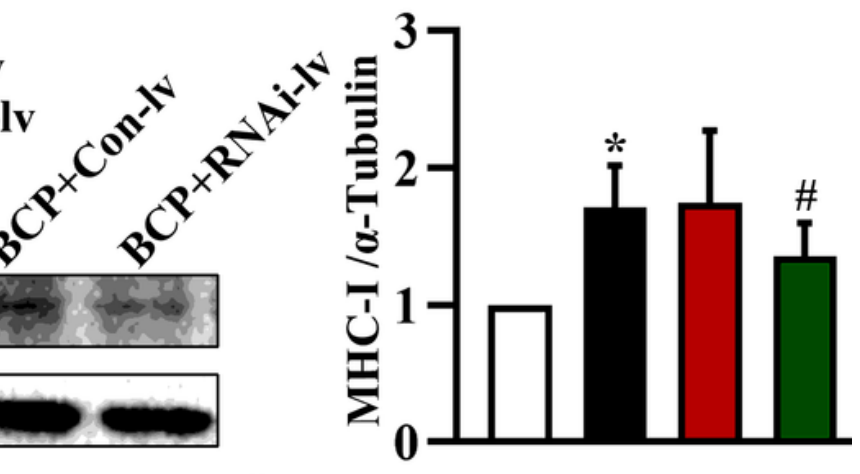

e

f
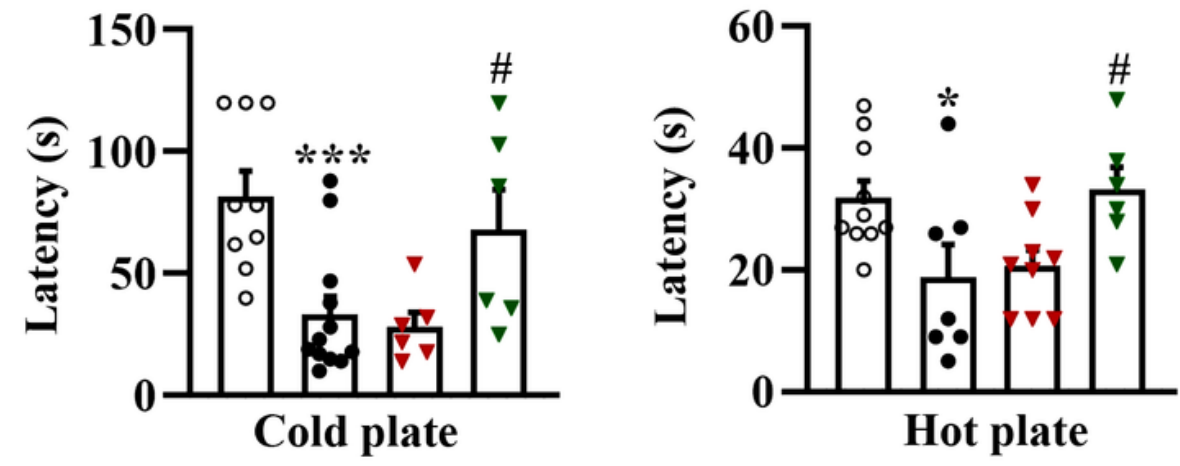

h

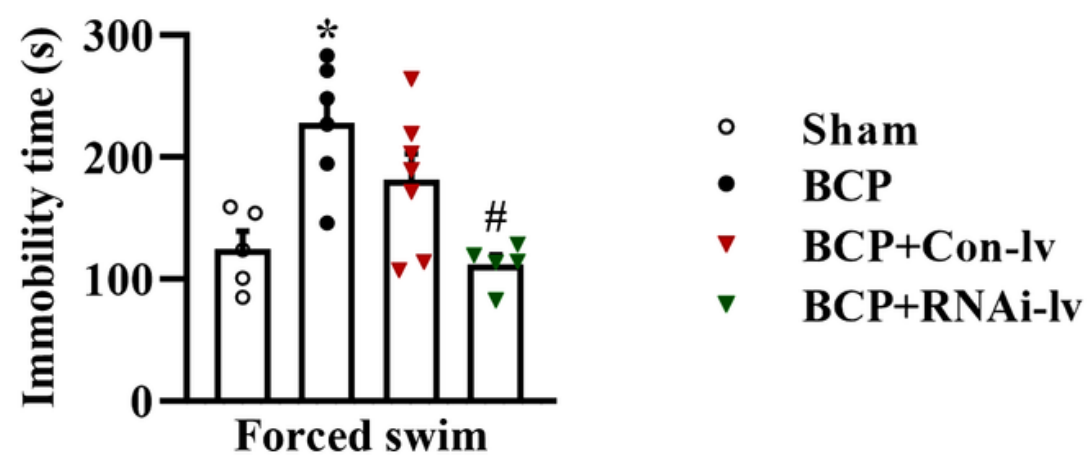

Figure 2

MHC-I knockdown attenuated mechanical hyperalgesia, heat- and cold-evoked allodynia, and depressivelike behavior in BCP mice. a. Representative immunofluorescence images of MHC-I interference lentivirus and control lentivirus injected into the hippocamous of BCP mice. EGFP (interference lentivirus or control lentivirus) labeled with MHC-I (magenta) revealed that MHC-I expression was markedly inhibited by interference lentivirus. Scale bar $=100 \mu \mathrm{m}$. b. The mRNA level of MHC-I was quantitative analysed by RTPCR among four groups. c. Representative immunoblots and quantitative analysis of MHC-I protein 
expression at day 21 after surgery in four groups, and a-Tubulin was used as an internal control. Values are normalized to the sham group and represented as mean \pm SEM. $n=4$ mice per group. ${ }^{*} P<0.05$ vs. sham group, ${ }^{\star \star \star} \mathrm{P}<0.001$ vs. sham group, $\# \mathrm{P}<0.05$ vs. BCP+Con-Iv group. $\mathrm{d}-\mathrm{f}$. Mechanical hyperalgesia of PWT (left), latency in cold plate test (middle), and latency in hot plate test (right) in four groups. Note that BCP mice exhibit decreased mechanical sensitivity and increased latency in hot and cold allodynia after tumor inoculation compared to sham mice, but MHC-I knockdown prevented these behaviors. $\mathrm{g}$-h. MHC-I knockdown decreased the time immobile in forced swim test (left) and tail suspension test (middle) on BCP mice. Data are represented as mean \pm SEM. ${ }^{*} \mathrm{P}<0.05$ vs. sham group, $\# \mathrm{P}<0.05$ vs. $\mathrm{BCP}+$ Con-lv group. 


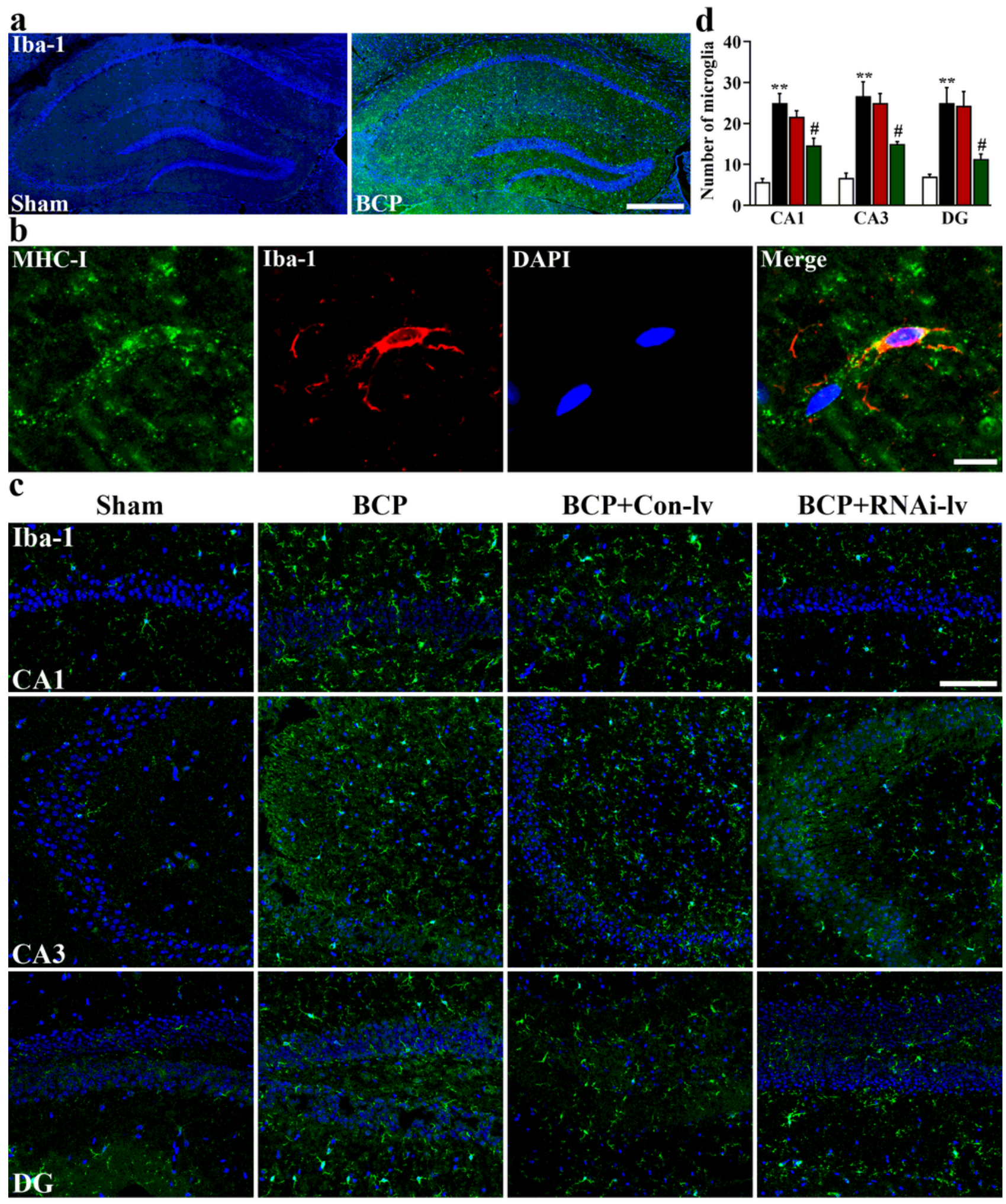

\section{Figure 3}

MHC-I knockdown reduced significant microglia activation in the hippocampus of BCP mice. a.

Representative immunofluorescence images of lba-1 (green) and DAPI (blue) in the dorsal hippocampus from sham and BCP mice on day 21 after surgey. Scale bar $=200 \mu \mathrm{m}$. b. Representative image of colocalization of MHC-I (green), Iba-1(red) and DAPI (blue). Scale bar $=10 \mu \mathrm{m}$. c. Images of Iba-1 (green) and DAPI (blue) in the CA1, CA3, and DG area of hippocampus from different groups. Scale bar=200 $\mu \mathrm{m}$. 
d. Quantification of microglia in CA1, CA3, and DG regions from Figure $3 \mathrm{C}$ among four groups. Values represent the mean \pm SEM in three independent experiments. $n=3$ mice per group. ${ }^{\star \star} P<0.05$ vs. sham group, \#P $<0.05$ vs. BCP+Con-Iv group.

a

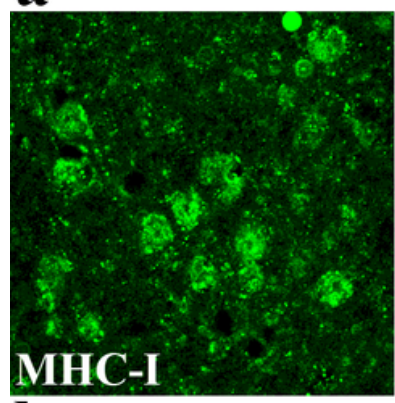

b
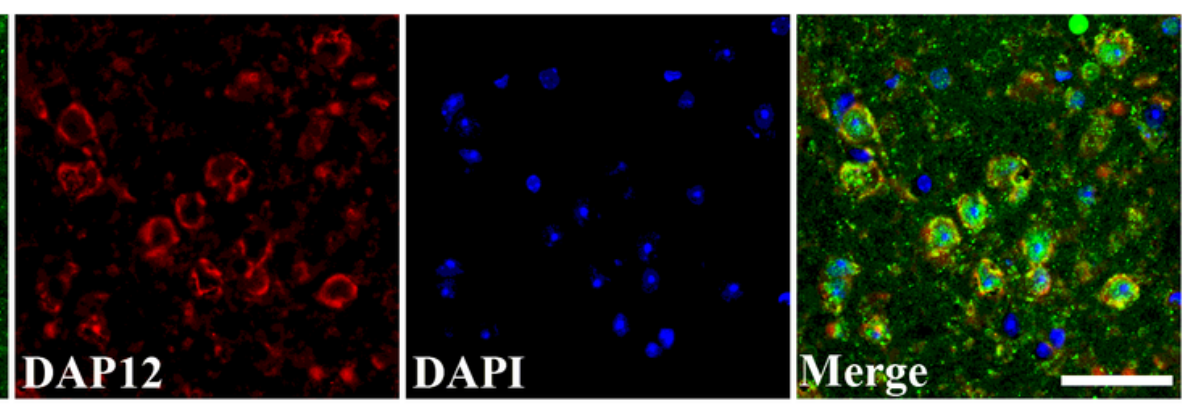

שSham

BCP

BCP+Con-lv

BCP+RNAi-lv

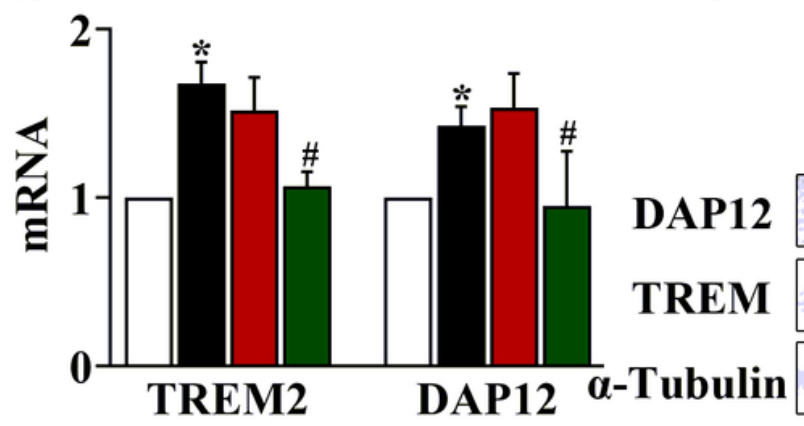

c

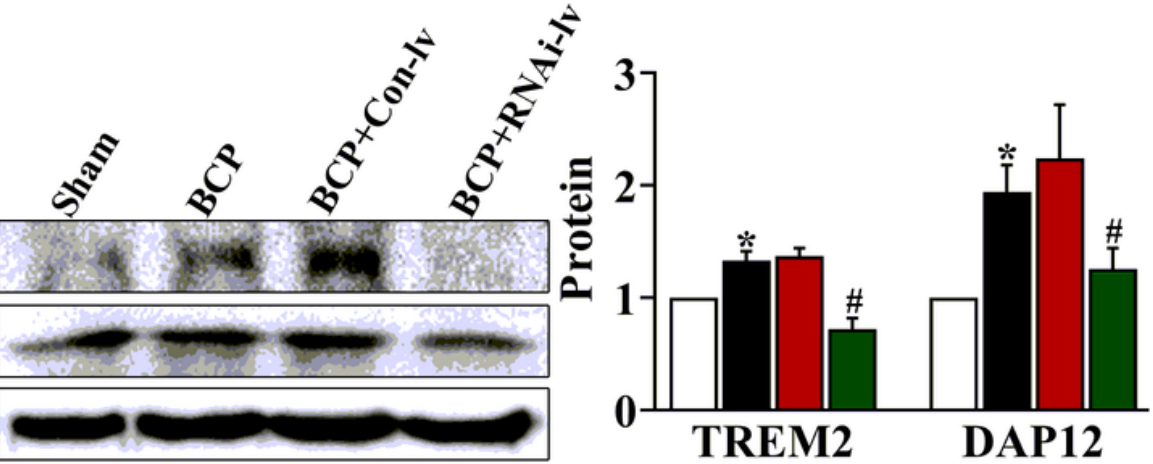

Figure 4

MHC-I knockdown decreased the level of TREM2 and DAP12 in hippocampus of BCP mice. a.

Representative immunofluorescence images of colocalization of MHC-I (green), DAP12 (red) and DAPI (blue) in hippocampus. Scale bar $=50 \mu \mathrm{m}$. b. The mRNA level of TREM2 and DAP12 were quantitative analysed by RT-PCR among four groups. c. Representative immunoblots and quantitative analysis of TREM2 and DAP12 protein expression at day 21 after surgery in four groups, and a-Tubulin was used as an internal control. Values are normalized to the sham group and represented as mean \pm SEM. $n=4$ mice per group. ${ }^{*} \mathrm{P}<0.05$ vs. sham group, $\# \mathrm{P}<0.05$ vs. $\mathrm{BCP}+$ Con-lv group. 